\title{
Cleft closure and undersizing annuloplasty improve mitral repair in atrioventricular canal defects
}

\author{
Muralidhar Padala, BS, ${ }^{a}$ Nikolay V. Vasilyev, MD, ${ }^{\mathrm{b}}$ James W. Owen, Jr, BS, ${ }^{\mathrm{a}}$ Jorge H. Jimenez, PhD, ${ }^{\mathrm{a}}$ Lakshmi P. Dasi, PhD, ${ }^{\mathrm{a}}$ \\ Pedro J. del Nido, MD, ${ }^{b}$ and Ajit P. Yoganathan, $\mathrm{PhD}^{\mathrm{a}}$
}

\begin{abstract}
Objective: Reoperation rates to correct left atrioventricular valve regurgitation after primary repair of atrioventricular canal defects remain relatively high. The causes of valvular regurgitation are likely multifactorial, and simple cleft closure is often insufficient to prevent recurrence.
\end{abstract}

\begin{abstract}
Methods: To elucidate the mechanisms leading to regurgitation, we conducted hemodynamic studies using isolated native mitral valves. Anatomy of these valves was altered to mimic atrioventricular canal type valves and studied under pediatric hemodynamic conditions. The impact of subvalvular geometry, cleft closure, annular dilatation, and annular undersizing on regurgitation were investigated.
\end{abstract}

Results: Papillary muscle position did not have a significant effect on regurgitation. Cleft closure had a significant impact on valvular competence, with reduction in regurgitation volume with increased cleft closure. Regurgitation volume decreased from $12.5 \pm 2.4 \mathrm{~mL} /$ beat for an open cleft to $4.9 \pm 1.9 \mathrm{~mL} /$ beat for a partially closed cleft and to $1.4 \pm 1.6 \mathrm{~mL} /$ beat when the cleft was completely closed. Annular dilatation had a significant impact on regurgitation even after cleft closure. A $40 \%$ increase in annular size increased regurgitation by $59 \%$ for a partially closed cleft and by $84 \%$ for a fully closed cleft. Reducing the annular size by $20 \%$ from the physiologic level decreased the regurgitation volume by $12 \%$ for a fully open cleft and by $58 \%$ for the partially closed cleft case.

Conclusions: Annular dilatation after primary repair has a potentially significant role in the recurrence of atrioventricular valve regurgitation. Reducing the annular size and restricting dilatation as an adjunct to cleft closure is a promising surgical approach in such valve anatomies.

In the current era of surgical repair for congenital atrioventricular canal (AVC) defects, the measure of operative success has shifted from early mortality to freedom from reoperation to correct residual lesions. In these patients, repair of the septal defects has been highly successful, but progressive postrepair regurgitation across the left atrioventricular (LAV) valve still remains a challenging problem. More than $15 \%$ of the patients surviving AVC repairs have hemodynamically significant regurgitation, the causes of which are unknown. ${ }^{1-4}$ Poor understanding of the underlying mechanisms causing postrepair LAV valve regurgitation has impeded progress toward optimizing valve repair in these patients. ${ }^{5}$

Unlike repair for other valve lesions, surgical guidelines for LAV valve reconstruction in AVC defects are unclear, resulting in evolution of a variety of unsuccessful repair procedures that often result in valve replacement. ${ }^{2,6,7}$ Longterm follow-up studies on these patients are scarce, so that

\footnotetext{
From the Wallace H. Coulter Department of Biomedical Engineering, Georgia Institute of Technology, Atlanta, Ga, ${ }^{\mathrm{a}}$ and the Department of Cardiac Surgery, Children's Hospital at Boston, Boston, Mass. ${ }^{\text {b }}$

Received for publication Jan 29, 2008; revisions received April 8, 2008; accepted for publication May 16, 2008.

Address for reprints: Ajit P. Yoganathan, PhD, Wallace H. Coulter Distinguished Faculty Chair in Biomedical Engineering and Regents Professor, Wallace H. Coulter School of Biomedical Engineering, Georgia Institute of Technology, Room 2119 U. A. Whitaker Building, 313 Ferst Dr, Atlanta, GA 30332-0535 (E-mail: ajit. yoganathan@bme.gatech.edu).

J Thorac Cardiovasc Surg 2008;136:1243-9

$0022-5223 / \$ 34.00$

Copyright (C) 2008 by The American Association for Thoracic Surgery

doi:10.1016/j.jtcvs.2008.05.043
}

it is impossible to assess the surgical techniques or understand the failure mechanisms. Key surgical questions such as the optimal length of cleft closure, ${ }^{8,9}$ impact of abnormal ventricular geometry, ${ }^{8,10,11}$ and growth of valvular structures after the repair remain unanswered. With the increasing number of AVC repairs performed every year, there is an immediate need to optimize valve repair in these patients by understanding the LAV valve hemodynamics under controlled conditions. Large animal models are ideal for such studies, but creating congenital anatomies in animals is yet to be accomplished. In this study, we present a novel in vitro method using porcine mitral valves to investigate the role of preoperative valve geometry, surgical procedure, and postoperative geometry on hemodynamics of the LAV valve. In particular, we investigate the impact of cleft closure, ventricular geometry, postoperative annular dilatation, and annular undersizing on valvular regurgitation.

\section{METHOD \\ Valve Preparation}

Eight mitral valves (24-mm size) were extracted from fresh porcine hearts (female pigs aged 4-6 months, weighing 100-120 kg) obtained from the local abattoir. The geometry of these native valves was altered to mimic the anatomy and function of the pathologic LAV valve in AVC defects, as shown in Figure 1. The P2 segment of the posterior leaflet was resected and the free edges were approximated with 4-0 Prolene polypropylene sutures (Ethicon, Inc, Somerville, NJ) (Figure 1, $A-D$ ), mimicking the smaller triangular posterior leaflet in AVC-like valves ${ }^{8,10}$ shown in Figure 1, $E$. The valve was then sutured onto a semi-oval annulus, with the curved portion of the semi-oval forming the posterior annulus and the straight 


\section{Abbreviations and Acronyms \\ $\mathrm{AVC}=$ atrioventricular canal \\ $\mathrm{EOA}=$ effective orifice area \\ $\mathrm{LAV}=$ left atrioventricular \\ $\mathrm{PM}=$ papillary muscle}

part of the semi-oval forming the anterior annulus (Figure 1). The papillary muscles (PMs) were wrapped in Dacron fabric and mounted on an adjustable geared fixture that allowed PM displacement in the apical, posterior, and lateral directions. ${ }^{12}$ The valves were then mounted into the in vitro experimental setup.

\section{In Vitro Experimental Setup}

The in vitro experimental setup used in this study is widely established and has been used in various studies on ischemic and degenerative mitral valve repairs. ${ }^{12-14}$ In brief, the left heart simulator (Figure 2, $A$ ) is a computer-controlled, pressure-driven, compressible bladder system that can simulate various pulsatile hemodynamic conditions of the left heart. It has a semi-oval silicone mitral annulus, whose size can be adjusted to desired levels (18-48 $\mathrm{mm}$ valve sizes) using a drawstring mechanism. This mechanism allows for segmental annular reduction and dilatation of the posterior annulus, keeping the anterior annulus at a constant length. The PMs are mounted onto an adjustable fixture that allows spatial displacement in the apical-basal, posterior-anterior, and lateral dimensions. Saline solution, $0.9 \% \mathrm{vol} / \mathrm{vol}$, as the working media and the chamber pressure and flow curves are monitored and recorded in real time with a differential pressure transducer (DP9-40; Validyne Engineering, Northridge, Calif), an electromagnetic flow probe (600 Series; Braemar Inc, St Paul, Minn), and a data acquisition system (DAQCARD 1200; National Instruments, Austin, Tex).

\section{Experimental Protocol}

All 8 valves were studied under the following experimental conditions: Baseline (control) conditions. Before the valve geometry was altered to an AVC-like valve, all the valves were studied under pediatric hemodynamic conditions: 120 beats/min heart rate, $85 \mathrm{~mm} \mathrm{Hg}$ peak left ventricular pressure, and an average cardiac output of $2.5 \mathrm{~L} / \mathrm{min}$. Annular size was maintained at the normal valve size (measured before valve extraction from the heart) with the PMs in their normal positions. The normal position of the PMs was determined such that the commissural chordae were in a single plane and the tips of the PMs were in the plane passing through the leaflet coaptation.

Disease conditions. The native valve geometry was then altered to mimic the AVC-like valve (Figure 1), and the experiments were repeated under the same pediatric hemodynamic conditions. First, the effect of abnormal ventricular geometry was studied with the PMs in their normal position (baseline) and by displacing them by $10 \mathrm{~mm}$ in the posterior and lateral directions (Figure 1, F), mimicking a scooped ventricular geometry found in these patients. After this step, a wedge-shaped anterior leaflet cleft was created by removing a triangular section of the anterior leaflet skirt from the free edge to the mitral annulus, as illustrated in Figure 1, $F$. The oblique PM position was maintained hereafter.

\section{Repair and postrepair conditions}

Cleft closure. The valve was repaired by closing the cleft to four levels: fully open cleft, one-third closed cleft, two-thirds closed cleft, and fully closed cleft, as shown in Figure 2, $B$. The total cleft length was measured in each valve and divided into three equal parts to determine the above cleft closure levels. Then, 4-0 Prolene polypropylene sutures were used to close the cleft in an interrupted knot fashion, and the valves were studied under pediatric hemodynamic conditions as previously used.
Annular dilatation. The impact of annular dilatation after cleft closure was studied by dilating the annulus to $20 \%$ and $40 \%$ of its normal area. In all the valves, the annular area was $4.0 \mathrm{~cm}^{2}$ initially and was increased to 4.8 $\mathrm{cm}^{2}$ to mimic $20 \%$ dilatation and to $5.6 \mathrm{~cm}^{2}$ for the $40 \%$ dilated size. These two levels of dilatation represent the mitral annular growth from infancy to 5 years of age, assuming the repair was conducted during infancy. ${ }^{15}$ The effect of annular dilatation was studied on the four cleft closure lengths.

Annular undersizing. The efficacy of annular undersizing as an adjunct procedure was investigated by undersizing the annulus from its normal size of $4.0 \mathrm{~cm}^{2}$ to $3.2 \mathrm{~cm}^{2}$, simulating $20 \%$ undersizing, and to $2.4 \mathrm{~cm}^{2}$, simulating $40 \%$ undersizing. The effect of undersizing was studied on the four cleft closure lengths.

\section{Data Acquisition and Measured End Points}

For all the experimental conditions, the transmitral and left ventricular pressures and the transmitral flow rate were recorded and the following end points were calculated:

Regurgitation volume. The transmitral flow curve was averaged over 15 cardiac cycles and the negative part of the flow curve was integrated to obtain the regurgitation volume per beat.

Effective orifice area (EOA). To assess valve stenosis, we calculated the diastolic EOA for each valve under all experimental conditions using the modified Gorlin equation: $\mathrm{EOA}=\mathrm{Q}_{\mathrm{rms}} /\left(51.6\left[\sqrt{ } \Delta \mathrm{P}_{\text {mean }}\right]\right)$ expressed in square centimeters, where $\mathrm{Q}_{\mathrm{rms}}$ (diastolic root mean square flow rate) and $\Delta \mathrm{P}_{\text {mean }}$ (mean diastolic transmitral pressure gradient) is used from averaged transmitral pressure and flow data.

Echocardiographic study. Three-dimensional echocardiographic measurements were obtained with an X7-2 pediatric 3-dimensional matrix array probe (iE33 System; Philips Medical Systems, Andover, Mass) at $3.75 \mathrm{MHz}$, at the highest possible frame rate at a sampling depth of 12 $\mathrm{cm}$. Two-dimensional echoes along the apical long-axis view cutting across both the leaflets anteroposteriorly at the midpoint of the major axis of the annulus were also obtained. Echocardiographic and color doppler images were obtained to assess the forward flow and the leakage flow through the cleft and the coaptation regions.

\section{Statistical Analysis}

All the data are reported as mean \pm 1 standard deviation. The data were tested for normality by an Anderson-Darling test (MINITAB 15). Different experimental groups were compared by paired $t$ tests.

\section{RESULTS \\ Effect of Smaller Posterior Leaflet and Oblique PM Position}

The smaller posterior leaflet by itself without the cleft did not affect valve function, with statistically insignificant changes $(P=.12)$ between the normal $(0 \mathrm{~mL} /$ beat $)$ and AVC-like valves $(0.2 \pm 0.1 \mathrm{~mL} /$ beat). Oblique displacement of the PMs did not disturb valve function, with the regurgitation volumes of $0 \mathrm{~mL} /$ beat (normal PM position) versus 0.7 $\pm 0.3 \mathrm{~mL} /$ beat (oblique) at normal annular size. Similarly, at a $40 \%$ dilated annular size, the regurgitation volume was 9.5 \pm 2.3 (normal) versus $9.3 \pm 2.5 \mathrm{~mL} /$ beat (oblique) $(P=.77$ ).

\section{Length of Cleft Closure}

Figure 3 illustrates the comparison between regurgitation volumes for the four cleft closure levels with a normal size annulus. With the cleft fully open, a regurgitation volume of $12.56 \pm 2.4 \mathrm{~mL} /$ beat was obtained. One-third cleft closure 


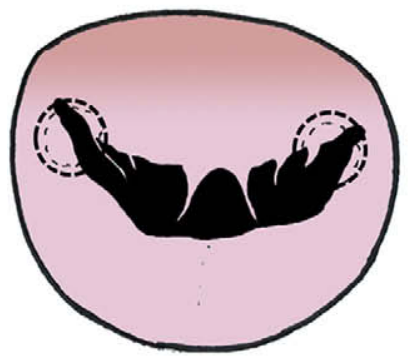

A

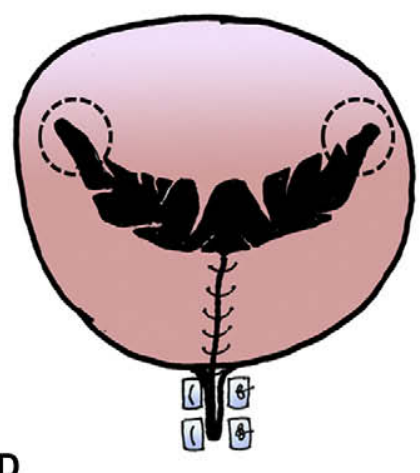

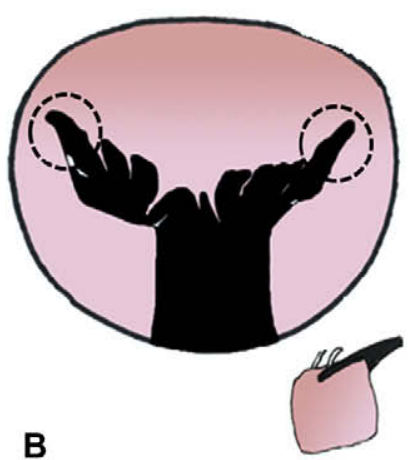

B

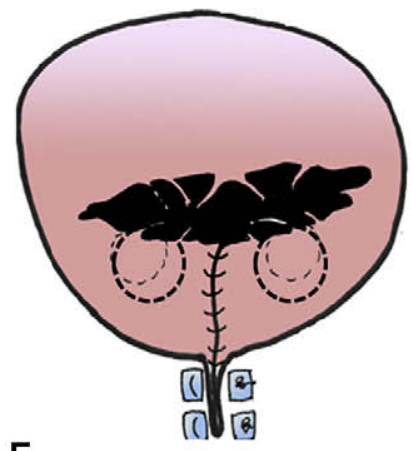

$\mathbf{E}$

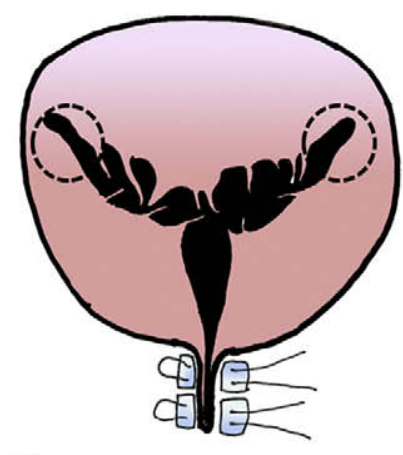

C

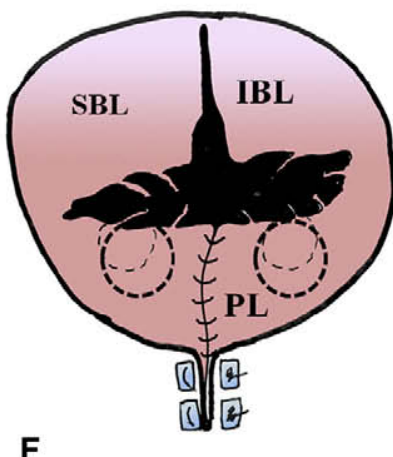

In-vivo 3D Echo of Valve Structure in a Patient
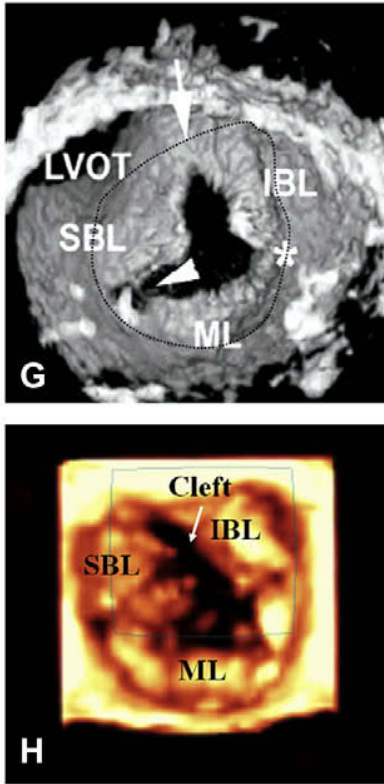

In-vitro 3D Echo of Valve Structure from our study

FIGURE 1. Sequential steps to alter the native mitral valve geometry to mimic the left atrioventricular valve in the canal defects. The dotted circles indicate the position of the papillary muscles. A, Native mitral valve. B, Resection of the P2 segment of the posterior leaflet. C, Plication of the annulus. D, The free ends of the resected section are sutured together, forming a smaller posterior leaflet. E, Posterior lateral displacement of the papillary muscles. F, The anterior leaflet skirt is sectioned to mimic the cleft. The images on the right $(\mathrm{G}$ and $\mathrm{H})$ compare the valve structure with cleft (arrow) obtained from our in vitro setup under pulsatile hemodynamic conditions with one obtained from a patient, using 3-dimensional echocardiography. $S B L$, Superior bridging leaflet; $I B L$, inferior bridging leaflet; $M L$, mural/posterior leaflet; $L V O T$, left ventricular outflow tract.

reduced it to $9.96 \pm 2.1 \mathrm{~mL} /$ beat and was significantly lower from the fully open cleft $(P=.001)$. A two-thirds cleft closure resulted in $4.9 \pm 1.9 \mathrm{~mL} / \mathrm{beat}(P=.001)$ and $1.4 \pm 1.6$ $\mathrm{mL} /$ beat $(P=.001)$ for complete cleft closure.

\section{Post-repair Annular Dilatation}

Annular dilatation significantly increased the regurgitant volume irrespective of the length of cleft closure, as shown in Figure 4, A. For a fully open cleft, the regurgitant volume increased from $12.2 \pm 2.1 \mathrm{~mL} /$ beat at normal annulus size to $13.7 \pm 1.1 \mathrm{~mL} /$ beat for $20 \%$ dilatation and $14.7 \pm 0.8 \mathrm{~mL} /$ beat for $40 \%$ dilatation. When a third of the cleft was closed, a $20 \%$ increase in the annular size increased the regurgitant volume to $12.1 \pm 2.4 \mathrm{~mL} /$ beat from $9.9 \pm 2.8 \mathrm{~mL} /$ beat at normal size $(P=.001)$ and to $14.0 \pm 1.7 \mathrm{~mL} /$ beat at $40 \%$ dilatation, which was significantly different from the normal annulus $(P=.002)$ and the $20 \%$ dilated annulus $(P=.007)$. For the two-thirds cleft closure, the regurgitant volume changed from $4.7 \pm 3.3 \mathrm{~mL} / \mathrm{beat}$ at normal annulus size to $8.5 \pm 2.5 \mathrm{~mL} /$ beat $(P=.002)$ and $11.4 \pm 1.8 \mathrm{~mL} /$ beat $(P$ $=.0001)$ for $20 \%$ and $40 \%$ annular dilatation, respectively.
Even when the cleft was fully closed, the regurgitant volumes increased from $1.4 \pm 2.8 \mathrm{~mL} /$ beat at normal annular size to $4.5 \pm 2.3 \mathrm{~mL} /$ beat $(P=.0001)$ for $20 \%$ dilatation and $8.7 \pm 1.5 \mathrm{~mL} /$ beat $(P=.0001)$ for $40 \%$ dilatation.

\section{Effect of Annular Undersizing on Regurgitation Volume}

Figure 4, $B$, illustrates the effect of undersizing the annulus on the regurgitant volume for the four cleft closure lengths. For the fully open cleft, regurgitation volume decreased from $12.56 \pm 2.4 \mathrm{~mL} /$ beat to $10.83 \pm 1.8 \mathrm{~mL} /$ beat for $20 \%$ undersizing and to $9.8 \pm 1.9 \mathrm{~mL} /$ beat for $40 \%$ annular undersizing $(P=.03)$. Similarly, for the one-third cleft closure length, $20 \%$ undersizing decreased regurgitant volume from $9.95 \pm 3 \mathrm{~mL} /$ beat to $7.78 \pm 2 \mathrm{~mL} /$ beat $(P=.007)$ and an additional $20 \%$ undersizing reduced it to $4.44 \pm 1.9$ $\mathrm{mL} /$ beat $(P=.001)$. For the two-thirds cleft closure length, the regurgitation volume decreased from $4.73 \pm 2.8 \mathrm{~mL} /$ beat at the normal annular size to $2.05 \pm 2.1 \mathrm{~mL} /$ beat $(P=.518)$ for $20 \%$ undersizing and to $0.7 \pm 1.2 \mathrm{~mL} /$ beat $(P=.533)$ for $40 \%$ undersizing. The $P$ values are in comparison with 
In-vitro Experimental Model
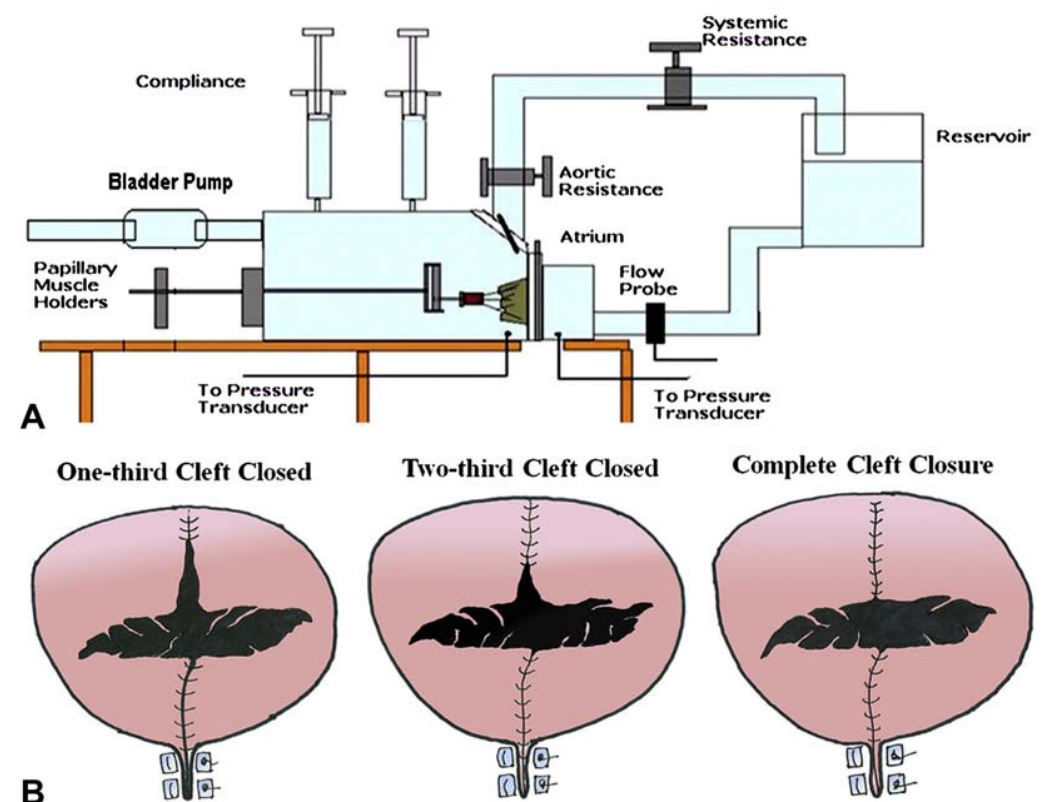

FIGURE 2. A, The schematic of the in vitro left heart simulator shows a native porcine mitral valve mounted into the simulator with the various components of the system labeled. B, The three different lengths of cleft closure that were studied.

the regurgitation volume of the valve with a smaller posterior leaflet and obliquely placed PMs, and without an anterior cleft.

\section{Diastolic EOA—Prerepair and Postrepair Conditions}

For all the valves, diastolic EOA was calculated before and after the repair, and the results are illustrated in Figure 5. No statistically significant changes were observed between the preoperative and postoperative groups $(P=.734)$, suggesting absence of valve stenosis even after annular undersizing. Small increases in the effective orifice areas were observed where the cleft was partially open.

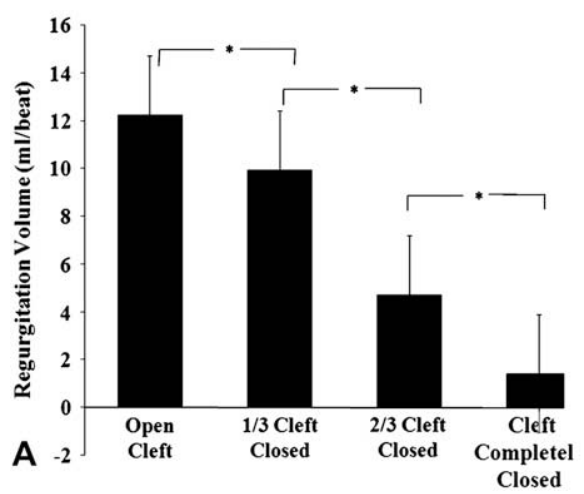

\section{DISCUSSION}

Clinical outcomes after correction of AVC defects depend largely on the surgical procedure and postoperative LAV valve function. In this study, we demonstrate the benefit of complete cleft closure on LAV valve competence in an in vitro AVC-like valve model. These results agree with the clinical findings from Wetter and associates ${ }^{9}$ in their comprehensive study on 96 patients over a period of 13 years and confirm the benefits of cleft closure. Despite complete cleft closure, these patients had progressively increasing LAV valve regurgitation resulting in high reoperation rates. ${ }^{16-18}$ A critical component of this study demonstrates the impact of postoperative annular dilatation on the repair, and the

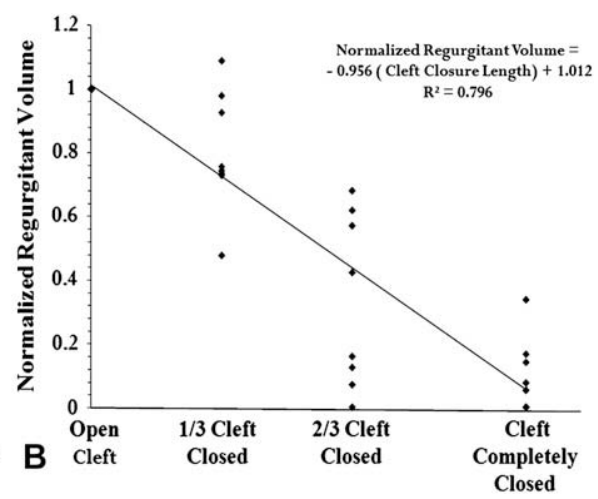

FIGURE 3. A, Regurgitation volumes obtained for open, one-third closed, two-thirds closed, and completely closed cleft lengths. B, Normalized regurgitation volume plotted against the cleft closure length for each valve. Normalized regurgitation volume was obtained by dividing the regurgitation volume for the one-third, two-thirds, and fully closed conditions with the corresponding open cleft regurgitation volume. The equation clearly shows a negative correlation between the regurgitation volume and the cleft closure length. 

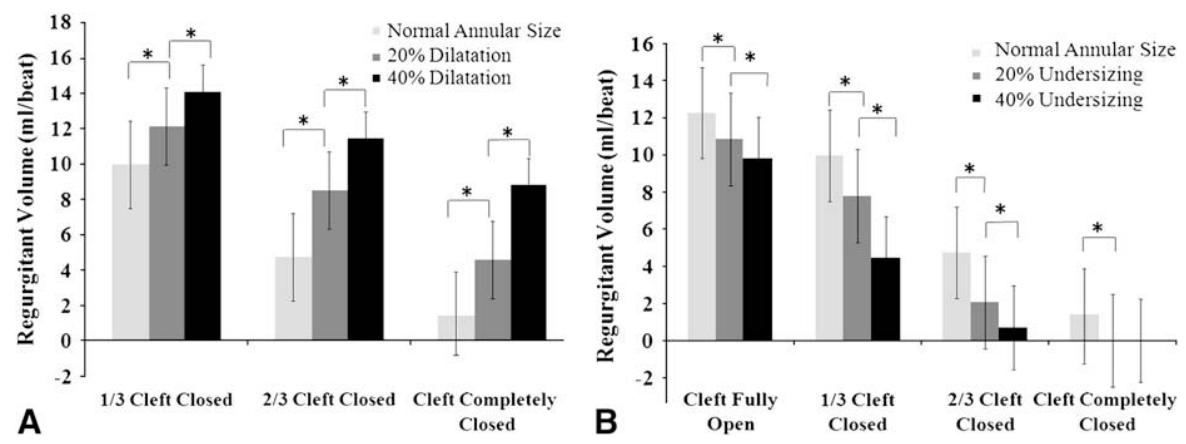

FIGURE 4. A, Increase in the systolic regurgitation volume owing to annular dilatation for the three levels of cleft closure. B, Decrease in the systolic regurgitation volume with $20 \%$ and $40 \%$ annular undersizing for the three levels of cleft closure.

results suggest that small magnitudes of postoperative annular dilatation induced in valve incompetence even after cleft closure, suggesting that isolated cleft closure is insufficient and the annulus has to be addressed as well.

To overcome the unavailability of large animal models, we adopted an in vitro method that simulates AVC-like valve anatomy and function, as shown in Figure $1, G$ and $H .^{1}{ }^{[1]}$. Although in vitro methodology is limited by its rigid ventricular chamber, nonphysiologic ventricular shape, lack of annular dynamics, and absence of chordal attachments from the ventricular wall, as seen in AVC patients, these limitations do not affect the variables that were investigated in this study. Additionally, the in vitro approach provides precise control over the valve geometry and hemodynamic conditions and also provides the ability to study different repair procedures on the same valve.

Previous clinical studies have reported that the key to competence of the trifoliate LAV valve is the zone of apposition (cleft) between its bridging leaflets. ${ }^{2,7,9}$ The findings from this study confirm these clinical observations and provide novel insights into the interaction between the degree of cleft opening and annular size after primary valve repair in AVC defects. Given the anatomic constraints in leaflet size

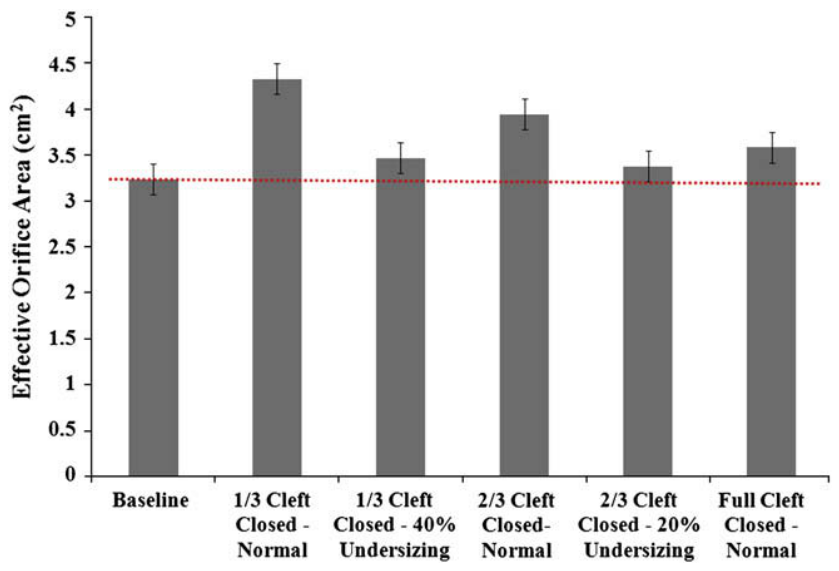

FIGURE 5. Comparison of the diastolic effective orifice areas obtained for the prerepair and postrepair conditions. and mobility in the AVC defect valves, such an understanding can aid surgical decision-making. The regurgitation volume measured in this study decreased by $18 \%$ for a one-third cleft closure, by $61 \%$ for a two-thirds closed cleft, and by $90 \%$ in the case of a completely closed cleft in comparison with a fully open cleft. These data suggest that if a partial cleft closure is intended, at least two thirds of the cleft should be closed to achieve hemodynamic benefits. Closing the cleft in the vicinity of the annulus leaves a majority of the cleft open, allowing regurgitation. When more than two thirds of the cleft is closed, regurgitation is prevented inasmuch as the small portion of the cleft that is left open extends into the leaflet coaptation. Often, mild regurgitation persists (Figure 6) through this region because the edges of the cleft are thin and unstable at high systolic pressures. Complete closure of the cleft restored excellent valve competence ( $91 \%$ reduction) with good leaflet mobility observed on the echo. However, complete closure of the cleft can potentially cause valvular stenosis in patients with a parachute valve or closely spaced PMs. Leaving the cleft partially open, in this group, might aid efficient diastolic left ventricular filling.

Although cleft closure greatly improves immediate surgical outcome, postrepair annular dilatation would have a significant negative impact on the long-term repair durability. Our results suggest that annular dilatation causes significant recurrence of regurgitation notwithstanding cleft closure. With a $20 \%$ increase in annular size, regurgitation volumes increased up to $45 \%$ for a two-thirds cleft closure and by $69 \%$ for complete cleft closure. At $40 \%$ annular dilatation, regurgitation increased by $59 \%$ for the two-thirds cleft closure and by $84 \%$ for the complete cleft closure. An interesting inference from these results is the higher gradient of increase in regurgitation resulting from annular dilatation for a fully closed cleft compared with a partially closed cleft. We speculate that for a complete cleft closure, chordae inserting into the anterior leaflet edges tether on the leaflets, which could be confounded by annular dilatation causing increased regurgitation confirmed by our color Doppler data in Figure 6. Normal mitral valve leaflets compensate up to 


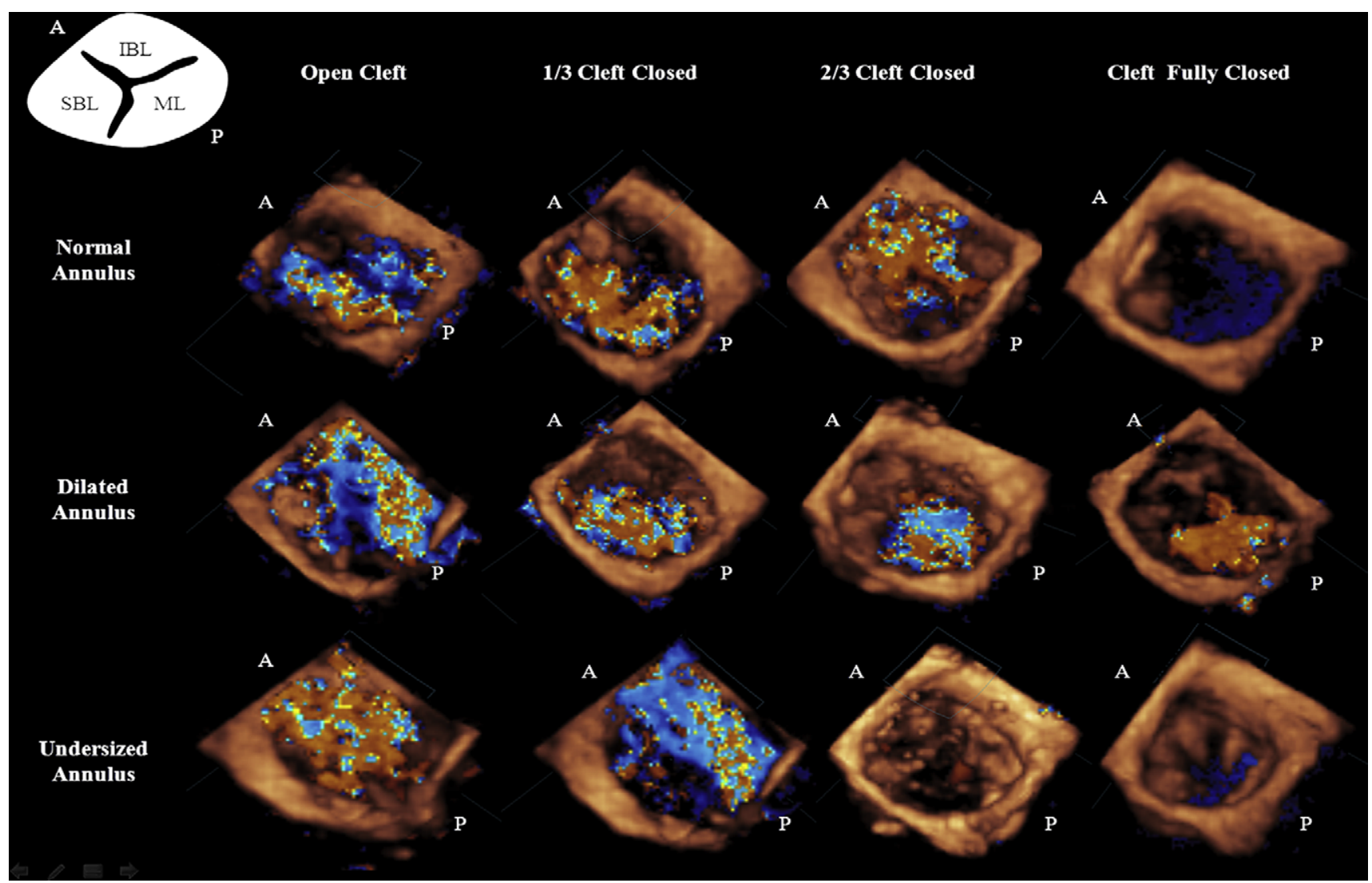

FIGURE 6. Three-dimensional full volume color Doppler images obtained at peak systolic valve closure. The images in the first row depict the reduction in the regurgitation volume with increasing cleft closure. The second row illustrates the recurrent regurgitation with dilatation of the annulus in all the cases of cleft closure. Images in the third row illustrate the efficacy of reducing the annular size in restoring valve coaptation even with a partial cleft closure. $A$, Anterior; $P$, posterior.

$80 \%$ annular dilatation before leaking. ${ }^{14}$ However, in these AVC-type valves, the posterior leaflet is nearly half the normal size, reducing the compensatory mechanisms. This important valve feature has to be acknowledged during valve repair. Posterior leaflet augmentation techniques could be beneficial in this scenario, but they are challenging to perform in infants owing to the fragility of leaflet tissue and the risk of cutting the chordae.

Annular undersizing may be a promising alternative to patch augmentation in these patients. In our study, we demonstrate that $20 \%$ annular undersizing with two-thirds cleft closure restored good valve competence by reducing the regurgitation by $58 \%$ from the normal annular size. A similar trend was observed with one-third cleft closure as well, but at $40 \%$ undersizing, which might cause valvular stenosis. Undersizing in these patients could serve three purposes: restrict annular dilatation, enhance leaflet coaptation, and restore complete valve competence with only a partial cleft closure. Inasmuch as AVC defects are corrected during infancy, restricting annular dilatation during the initial years after the repair could prevent late regurgitation. However, this procedure has to be considered cautiously, and adult mitral annuloplasty rings should not be used owing to their rigidity and lack of growth potential, potentially causing stenosis in the long term. Using polydioxanone or absorbable annuloplasty rings is recommended because they allow somatic growth of the annulus by gradual degradation with time. ${ }^{19}$ This strategy may attenuate early morbidity and also allow for better long-term valve growth and performance. This study also supports the hypothesis that a partial cleft closure is sufficient when undersizing the annulus. Acknowledging this fact is useful when confronting difficult anatomies such as in valves with a single PM. Additionally, the open part of the cleft could provide better leaflet mobility, ${ }^{10}$ benefiting diastolic ventricular filling.

Although the in vitro model adopted in this study simulates human AVC-like valve anatomy and function to a good extent, it has some inherent limitations. In this model, chordal attachments in the cleft vicinity inserting from the ventricular septum (Rastelli type A) were not simulated. These chordal attachments could contribute to some extent to the postoperative regurgitation. The rigid ventricular chamber used in this study does not mimic the ventricular motion, whose effects on the valve function were not studied. 
In summary, this study provides novel mechanistic insights into a complex problem of LAV valve repair in AVC defects. Cleft closure and postoperative annular dilatation have a significant impact on repair outcome. To reduce reoperation rates and to maintain long-term valve competence, we demonstrate that cleft closure with annular undersizing is proven to be beneficial.

We acknowledge Holifield Farms, Covington, Georgia, for donating porcine hearts that were used in this study.

\section{References}

1. Takahashi K, Guerra V, Roman KS, Nii M, Redington A, Smallhorn JF. Threedimensional echocardiography improves the understanding of the mechanisms and site of left atrioventricular valve regurgitation in atrioventricular septal defect. J Am Soc Echocardiogr. 2006;19:1502-10.

2. Murashita T, Kubota T, Oba J, Aoki T, Matano J, Yasuda K. Left atrioventricular valve regurgitation after repair of incomplete atrioventricular septal defect. Ann Thorac Surg. 2004;77:2157-62.

3. Najm HK, Endo M, Stephens D, Rebeyka IM, Williams WG. Complete atrioventricular septal defects. Results of repair, risk factors and freedom from reoperation. Circulation. 1997;96:311-5

4. Bando K, Turrentine MW, Sun K, Sharp TG, Ensing GJ, Miller AP, et al. Surgical management of complete atrioventricular septal defects: a twenty-year experience. J Thorac Cardiovasc Surg. 1995;110:1543-52; discussion 1552-4.

5. Tlaskal T, Hucin B, Marek J, Chaloupecky V, Kostelka M, Janousek J, et al. Individualized repair of the left atrioventricular valve in spectrum of atrioventricular septal defect. J Cardiovasc Surg (Torino). 1997;38:233-9.

6. Michielon G, Stellin G, Rizzoli G, Milanesi O, Rubino M, Moreolo GS, et al. Left atrioventricular valve incompetence after repair of common atrioventricular canal defects. Ann Thorac Surg. 1995;60(6 Suppl):S604-9.

7. Ten Harkel AD, Cromme-Dijkhuis AH, Heinerman BC, Hop WC, Bogers AJ. Development of left atrioventricular valve regurgitation after correction of atrioventricular septal defect. Ann Thorac Surg. 2005;79:607-12.
8. Anderson RH, Zuberbuhler JR, Penkoske PA, Neches WH. Of clefts, commissures, and things. J Thorac Cardiovasc Surg. 1985;90:605-10.

9. Wetter J, Sinzobahamvya N, Blaschczok C, Brecher AM, Gravinghoff LM, Schmaltz AA, et al. Closure of the zone of apposition at correction of complete atrioventricular septal defect improves outcome. Eur J Cardiothorac Surg. 2000;17:146-53.

10. Kanani M, Elliott M, Cook A, Juraszek A, Devine W, Anderson RH. Late incompetence of the left atrioventricular valve after repair of atrioventricular septal defects: the morphologic perspective. J Thorac Cardiovasc Surg. 2006;132:640-6, 646.e1-3.

11. Anderson RH, Neches WH, Zuberbuhler JR, Penkoske PA. Scooping of the ventricular septum in atrioventricular septal defect. J Thorac Cardiovasc Surg. 1988; 95:146.

12. Jimenez JH, Soerensen DD, He Z, Ritchie J, Yoganathan AP. Mitral valve function and chordal force distribution using a flexible annulus model: an in-vitro study. Ann Biomed Eng. 2005;33:557-66.

13. He S, Fontaine AA, Schwammenthal E, Yoganathan AP, Levine RA. Integrated mechanism for functional mitral regurgitation: leaflet restriction versus coapting force: in-vitro studies. Circulation. 1997;96:1826-34.

14. He S, Lemmon JD Jr, Weston MW, Jensen MO, Levine RA, Yoganathan AP. Mitral valve compensation for annular dilatation: in-vitro study into the mechanisms of functional mitral regurgitation with an adjustable annulus model. J Heart Valve Dis. 1999;8:294-302.

15. Behrman RE, Jenson HB. Nelson textbook of pediatrics. Philadelphia: Elsevier; 2007.

16. Rammohan MR, Bhan SA, Airan B, Juneja R, Saxena A, Kothari SS, et al. Routine cleft closure in repair of complete atrioventricular septal defects. Indian Heart J. 1998;50:527-30.

17. Suzuki K, Tatsuno K, Kikuchi T, Mimori S. Predisposing factors of valve regurgitation in complete atrioventricular septal defect. J Am Coll Cardiol. 1998;32. 1449-53.

18. Moran AM, Daebritz S, Keane JF, Mayer JE. Surgical management of mitral regurgitation after repair of endocardial cushion defects: early and midterm results. Circulation. 2000;102(19 Suppl 3):III160-5.

19. Miyamura H, Eguchi S, Watanabe H, Kanazawa H, Sugawara M, Tatebe S, et al. Total circular annuloplasty with absorbable suture for the repair of left atrioventricular valve regurgitation in atrioventricular septal defect. J Thorac Cardiovasc Surg. 1994;107:1428-31. 